\author{
EVS27 \\ Barcelona, Spain, November 17-20, 2013
}

\title{
Experimental Setup to Explore the Drives of Battery Electric Vehicles
}

\author{
Anton Rassõlkin, Valery Vodovozov \\ Electrical Engineering Department, Tallinn University of Technology, 19086, Tallinn, Ehitajate tee 5, Estonia \\ Anton.Rassolkin@ttu.ee, Valery.Vodovozov@ttu.ee
}

\begin{abstract}
This paper describes an experimental setup for research and exploring the drives of battery-fed electric vehicles. Effective setup composition and its components are discussed. With experimental setup described in this paper, durability and functional tests can be procured to the customers. Multiple experiments are performed in the form of steady-state system exploring, acceleration programs, multi-step tests (speed control, torque control), load collectives or close-to-reality driving tests (driving simulation). Main focus of the functional testing is on the measurements of power and energy efficiency and investigations in driving simulation mode, which are used for application purposes. In order to enable the examination of the drive trains beyond standard modes of operation, different other parameters can be studied also.
\end{abstract}

Keywords: BEV (battery electric vehicle), drive, energy consumption

\section{Introduction}

Automotive industry has turned into a primary market for electric drive and power electronic products. Accurate alternating current (ac) and direct current $(\mathrm{dc})$ motor drives over a wide range of power and speed fed by power converters based on insulated gate bipolar transistors with complex monitoring and management systems have become an inherent part of modern vehicles [1]. In this a context, exploring and test platforms of a battery-operated electric vehicle (BEV) fully propelled by electrical motors are drawing today significant attention. They allow to study and to optimise vehicle performance, to reduce the number of test runs of real machines, and to provide their safety. Many research institutions and an increasing number of engineering schools introduce test benches in their laboratories [2]. Severe references describe multiple kinds of experimental setups developed in different countries [3]-[10]. Most of them concern the energy management, optimal configuration, and proper combination of different energy sources of BEVs, such as batteries, supercapacitor packs, flywheels, fuel cells, etc.

Effective platforms used to study the drives of BEVs may serve as departments of the national electromobility centres. The results of their performance affect both the choice of the drive components and the assessment of the particular drive manufacturing technologies. Their hardware and software promote comparison the different models of the propulsion drives thus helping the community in selection the car models and companies for supporting and backing and providing customers with many hidden data of the marketable electric cars. Above all, they support BEV developers by reducing the design times and costs requirements in this field.

The platform described in this paper opens new possibilities in analyzing and comparison of BEV motor drives from the viewpoint of their power 
economical performance resulting in selection of BEVs most appropriate for regional road and climate conditions. The platform is suitable for exploring different steady-state and dynamic modes of the motor drive performance in multiple BEV applications. Therefore, this category of the simulation and experimental techniques is effectively applied in drives working in many BEV motor modes. As well the durability and functional tests can be requested from the test bench by the customers, including the following:

- depending on the respective prototype phase of the specimen, multiple tests can be performed in the form of acceleration block programs, multi-step tests (speed control, torque control), load collectives or close-to-reality driving tests (driving simulation)

- main focus of the functional testing is on the measurements of power and energy efficiency and investigations in driving simulation mode, which are used for application purposes

- for highly accurate power measurement, a pool of different torque measuring procedures is included in the platform that adopt the experimental setup and the measurement range to the tested physical values

- to enable an examination of the drive trains beyond standard modes of operation, different parameters like wheel slip (variable friction coefficient and adjustable rotational inertia of the simulated vehicle wheels), multiple wheel speed left/right, front/rear, and uphill/downhill grades may be simulated

General configuration of the BEV involves three major subsystems - electric propulsion, energy source, and auxiliary. The first subsystem comprises the power converter, electrical motors, mechanical transmission, and driving wheels. The energy source subsystem includes energy sources, energy management system, and energy refuelling unit. The auxiliary subsystem consists of the power steering unit, temperature control unit, and auxiliary power supply [11].

The motor drives, being the main components of BEVs, affect the efficiency, performances, and costs. This is the reason, why the BEV drives are of the main concern in this paper. The investigations are focused on the motors, power converters, supply chains, and transmissions, which are used in BEVs. The main objectives of the experimental setup development are as follows:
- to provide the research environment for analysis, investigation, and simulation of the marketable BEV drive systems

- to establish the assessment and verification procedures for different motor, gear, and power converter types met for propulsion

- to support commercial consulting, research and testing for enterprises

- to enlarge students' participation in corresponding research topics

The experimental setup involves the testing motor system, the simulated vehicle load, and the informational environment DriveWindow implemented on the basis of ACS 600/800 motor drives of ABB [12]. The system runs two motors, one working as a propulsion drive imitator and the other as a load imitator, with a physical coupling through the self-manufactured prototype of the cardan transmission.

Results obtained from the experimental setup can affect both the choice of the drive components and the assessment of the particular drive manufacturing technologies. They are suitable for comparison the different models of the propulsion drives from such points of view as their dynamic performance at start-up and braking, static stability on the road, energy consumption, reliability, and control suitability

\section{Considerations of Power Supply}

The major part of the powertrain of an experimental setup accomplishes two power sources that feed an electric motor propelling the $\mathrm{BEV}$, as shown in Figure 1.

The three-phase network supplies the dc power bus through a rectifier. Another power source should imitate an on-board energy system connected to the bus. A dc/dc converter allows the rectifier and the on-board source to have a different voltage and to adjust the power flow to fully use the energy. It boosts the battery output voltage up to the maximum dc bus voltage of $560 \mathrm{~V}$ feeding the traction motor. As soon as the accelerator pedal is released, a traction machine turned by wheels acts as a generator, which voltage is converter back by the inverter freewheeling diodes, and then the booster drops this voltage back to charge the battery.

A battery being the main supply of high specific energy, is optimized for the rout travelling. As the auxiliary supply, supercapacitors could be used. In the simplest configuration, both the main and the auxiliary supplies are connected in parallel. The system operates in three different modes: 
- traction, i.e., the motor provides a propulsion force to the $\mathrm{BEV}$;

- braking, i.e., the brakes dissipate kinetic energy of the BEV while the motor can be engaged or disengaged;

- coasting, i.e., the motor is disengaged and the resistant losses of the BEV are exactly matched be the decrease of its kinetic energy.

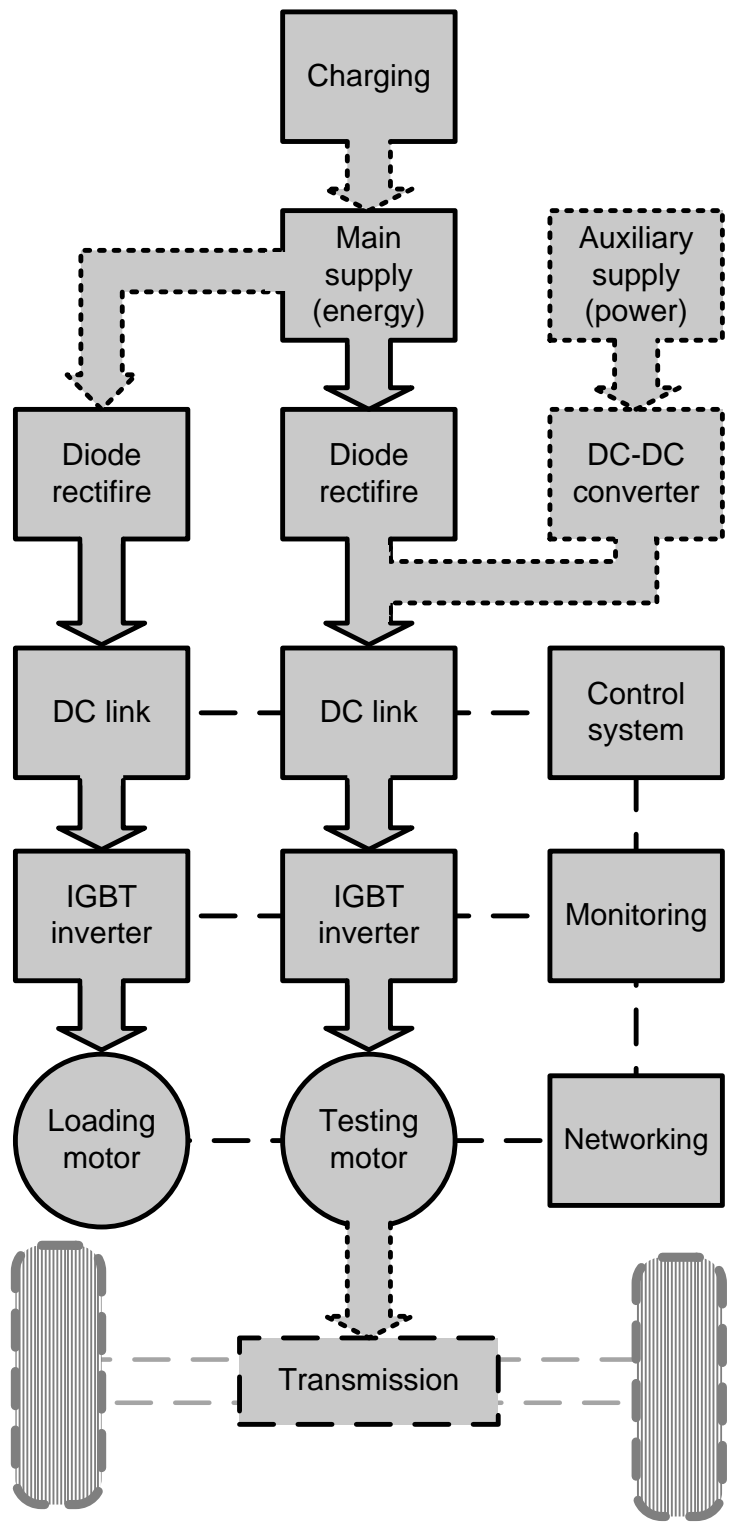

Figure 1: Powertrain experimental setup

During the cruising and coasting, the main supply feeds the drive to provide traction. To enable the system readiness for sudden power demand, this high specific energy source can precharge the high specific power source in the light-road period. During the acceleration or hillclimbing traction conditions, both the sources need to simultaneously supply the desired energy to the drive. During the braking or downhill condition, the drive operates as a generator so that the regenerative energy flows back to recharge the high specific power source. As the power flow cannot be actively controlled in this mode of operation, a two-quadrant dc/dc converter is placed between the main and the peak power sources. This design supports different voltages of the main supply and the peak power source and provides adjustable power flow between them.

To feed the electrical motor during the power demand rises or to recharge the batteries when power demands come down, the supercapacitor pack is used as the high specific peak power source. This energy storage implements high dynamics for adding energy to motors when a vehicle needs extra power for acceleration, to overcome heavy loads, and for the regenerative braking. While the supercapacitor specific power is much higher than in batteries, its specific energy is substantially lower. Since the available supercapacitors for BEV application are of relatively low voltage level, an additional dc-dc converter is used to interface between the main supply and the supercapacitor terminals.

In the future research, battery, flywheel, UPS and fuel cell applications can also be discussed for the experimental setup uninterruptible supply and as the main on-board supply source along with the rectifiers.

\section{Performance of experimental setup}

Experimental setup located in the Electrical Drives Laboratory of Tallinn University of Technology performs in static and dynamic testing modes [12]. To explore dynamics of the experimental setup, the standard urban driving cycle ECE-R15 is commonly taken, which represents an alternating speed of the BEV in the predefined time intervals.

The timing traces in Figure 2 shows the measured values of speed and power corresponding to the ECE-R15 set-point values. The measurements were taken by applying the constant rated load to the testing drive. The active power distribution during the driving cycle shows the power peaks at acceleration and power falls during deceleration. More careful study of power spikes could give tips for choosing peak power source (supercapacitor) parameters and parameters of the power electronics converter between main and peak power sources.

At the static operational mode of the experimental setup, a constant speed vehicle motion mode could 
be described and imitated by stable set-point values. For dynamic loads, the performance of the powertrain is more complicated and can be simplified by dividing the motion on some basic load modes.

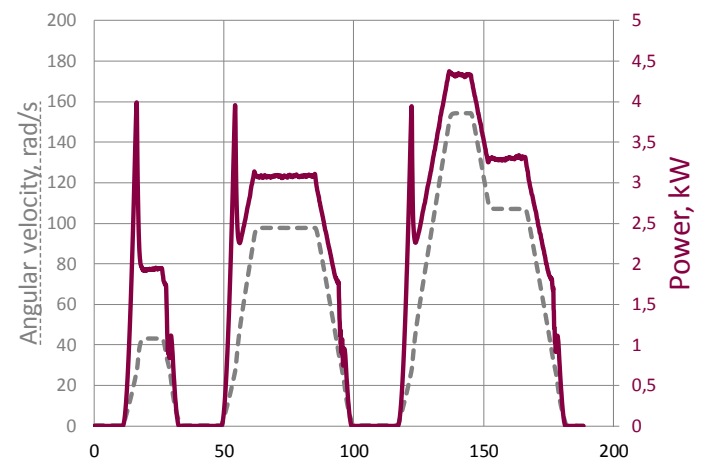

Figure 2: Angular velocity and active power timing diagram obtained from the experimental setup

Many main traction modes could be approximated as some simple shapes, such as meander, triangle, trapeze, and sine wave. By loading the testing drive of the experimental setup with such impacts, the following driving habits could be studied. A meander reference cycle imitates some typical parking regimes. A triangle reference cycle reference simulates uphill and downhill modes of the vehicle operation. A trapezoidal reference cycle is a composition of the triangle and meander, which imitates the most conventional city cruising regimes, particularly between the traffic lights. By a sinusoidal reference cycle, accurate travelling up the gradient and taking a turn can be effectively simulated. During the studies of the typical driving habits, the loading reference cycles presented in Figure 3 were applied to the testing drive.
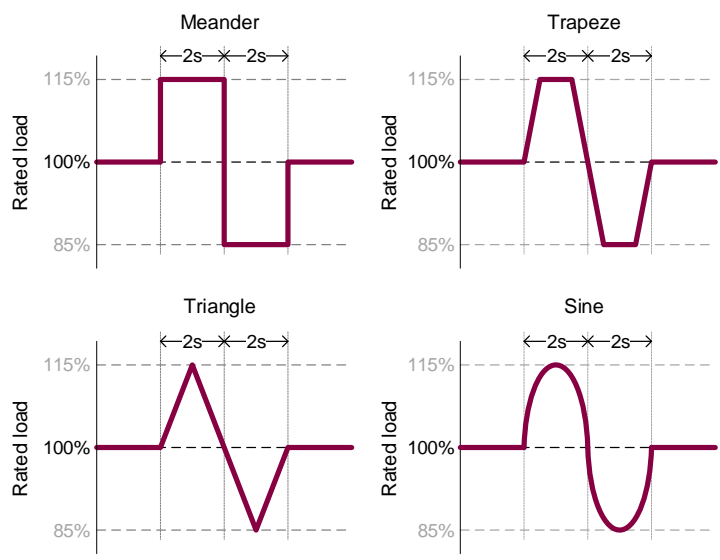

Figure 3: Loading reference cycles
Some examples of data observing from the experimental setup during applying the tests are presented in Figure 4. The tests were conducted in different control modes of the drive - the sensorless closed-loop (DTC) and the open-ended (scalar) modes. As it can be seen from the diagrams, the open-ended system smoothly responses on a load disturbance whereas the closed-loop system, due to feedback, more exactly copies the loading references. As the spikes and overlapping are not recommended for the slow energy storage devices, such as batteries, the use of additional peak power source could be recommended for the closed loop systems. At the same time, with an open-ended system the less loading of the battery during the same reference cycles can be observed.

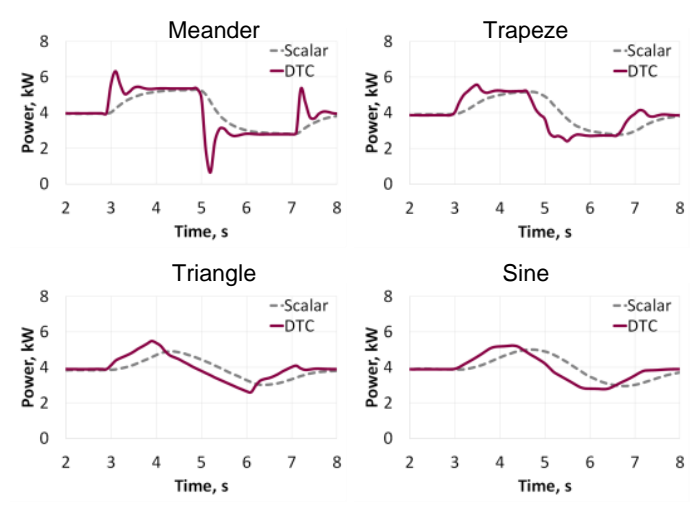

Figure 4: Active power observed from the experimental setup during applying the loading reference cycles

The energy consumption during the tests with the same loading references is presented in Figure 5. As it can be seen from the diagram, during the open-ended performance energy consumption in the meander and triangle reference cycles is less than during the closed-loop performance. As in the scalar operation, the motor speed is some below the reference, the energy is also less. An additional energy consumption in the DTC mode results from the feedback, which supports the motor speed according to the reference value all round the cycle. 


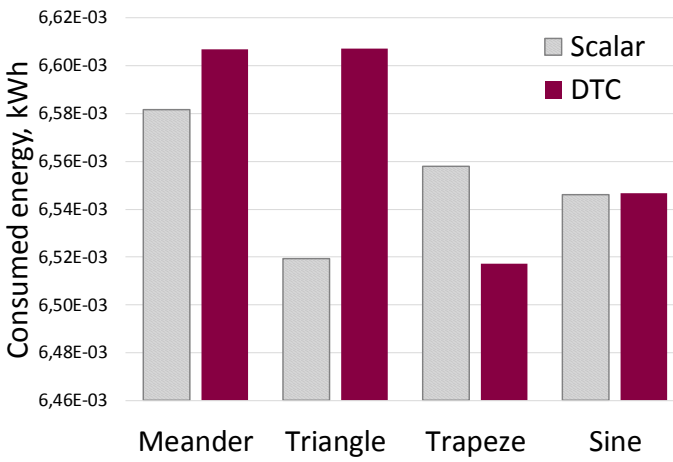

Figure 5: Energy consumption observed from the experimental setup during applying the loading reference cycles

During the sinusoidal reference cycle, the speed alternates more smoothly, and in that case the feedback does not have such effect on energy flow and energy consumption. The trapeze reference cycle represents a transient mode averaged between the triangle reference cycle and the sinusoidal reference cycle, in which the energy flow depends on the settings of the drive and the duty cycle.

The results obtained from the experimental setup show that, from the energy consumption point of view, the system works more cost effective in the case of mixed feedback, open-ended in some parts of the route and closed-loop in another.

\section{Conclusions}

With experimental setup described in this paper, durability and functional tests can be procured to the customers as well as many other problems can be solved, such as the following:

- testing and verification the functionality of the constituent equipment

- experimental determining of the mechanical characteristics of the propulsion motors and electrical properties of their power converters

- determining the charge/discharge characteristics of the supercapacitors

- determining the model of the traction motor and simulating the functionality of the system consisting of different combinations of energy sources and mechanical loads, in order to establish the optimal configurations for the system

- defining a procedure for an efficient management of the energy in the case of the functionality with unique sources and/or with hybrid sources (network, supercapacitors, etc.)
- studying the energy recovery processes and efficiency during the BEV braking processes Depending on the respective prototype phase of the specimen, multiple tests can be performed in the form of steady-state system exploring, acceleration programs, multi-step tests (speed control, torque control), load collectives or closeto-reality driving tests (driving simulation). Main focus of the functional testing is on the measurements of power and energy efficiency and investigations in driving simulation mode, which are used for application purposes. In order to enable the examination of the drive trains beyond standard modes of operation, different parameters like wheel slip, multiple wheel speed, left/right, front/rear, and uphill/downhill grades can be studied also.

\section{Acknowledgments}

This research has been supported by Estonian Ministry of Education and Research (Project SF0140016s11).

Publication of this paper has been supported by European Social Fund (project "Doctoral School of Energy and Geotechnology II").

\section{References}

[1] B. Fahimi and T. Sebastian, Guest editorial special section on automotive electromechanical converters, IEEE Transactions on Vehicular Technology, v. 56, n. 4, 2007, pp. 1470-1476.

[2] M. Ehsani, Y. Gao and A. Emadi, Modern Electric, Hybrid Electric, and Fuel Cell Vehicles: Fundamentals, Theory, and Design, CRC Press, Boca Raton, Florida, USA, 2010.

[3] C. Lungoci, D. Bouquain, A. Miraoui and E. Helerea, Modular test bench for a hybrid electric vehicle with multiples energy sources, 11th International Conference on Optimization of Electrical and Electronic Equipment OPTIM 2008, "Transilvania" University of Brasov, Brasov, Romania, 2008. pp. 299-306.

[4] Test Facilities for Automotive Research and Development, IKA Institut für Kraftfahrzeuge, RWTH Aachen University, FKA Forschungsgesellschaft Kraftfahrwesen $\mathrm{mbH}$, Aachen, Germany, 2011, 44 p.

[5] Y. Cheng, V-M. Joeri and P. Lataire, Research and test platform for hybrid electric vehicle with the super capacitor based energy storage, European Conference on Power Electronics 
and Applications EPE 2007, Aalborg, Denmark, 2007, pp. 1-10.

[6] P. Khatun, C. M. Bingham, N. Schofield and P. H. Mellor, An experimental laboratory bench setup to study electric vehicle antilock braking/traction systems and their control, IEEE 56th Vehicular Technology Conference VTC 2002, Vancouver, Canada, 2002, pp. 1490-1494.

[7] L. Jun, W. Li-fang, Y. Jian and L. Gui-dong, Research of a novel flexible load for electric vehicle test bench, International Conference on Computer and Communication Technologies in Agriculture Engineering CCTAE 2010, Chengdu, China, 2010, pp. 223-226.

[8] F. Marra, D. Sacchetti, A. B. Pedersen, P. B. Andersen, C. Træholt and E. Larsen, Implementation of an electric vehicle test bed controlled by a virtual power plant for contributing to regulating power reserves, 2012 IEEE Power \& Energy Society General Meeting, San Diego, USA, 2012, pp. 1-7.

[9] I. Alcala, A. Claudio and G. Guerrero, Test bench to emulate an electric vehicle through equivalent inertia and machine dc, 11th IEEE International Power Electronics Congress CIEP 2008, Cuernavaca, Mexico, 2008, pp. 198-203.

[10] Z. Hui, L. Cheng and Z. Guojiang, Design of a versatile test bench for hybrid electric vehicles, IEEE Vehicle Power and Propulsion Conference VPPC 2008, Harbin, China, 2008, pp. 1-4.

[11] V. Vodovozov, Z. Raud and T. Lehtla, A toolbox to design inverters for automotive applications, 11th World Conference on Applications of Electrical Engineering AEE 2012, Vouliagmeni, Athens, Greece, 2012, pp. 190-195.
[12] A. Rassõlkin and V. Vodovozov, A test bench to study propulsion drives of electric vehicles, 8th International Conference-Workshop Compatibility and Power Electronics CPE 2013, Ljubljana, Slovenia, 2013, pp. 275 $-279$.

\section{Authors}

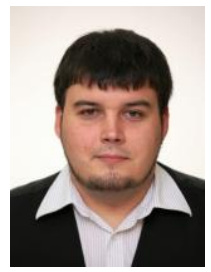
M.S. degrees in electric drives and power electronics from Tallinn University of Technology, Tallinn, Estonia, in 2008 and 2010, respectively. In 2010 received Dipl.Eng. (FH) degree in automatics from University of Applied Science Giessen-Friedberg, Giessen, Germany. Main scientific interests lay in the electric transportation. Since 2010 he is a PhD student at Tallinn University of Technology.

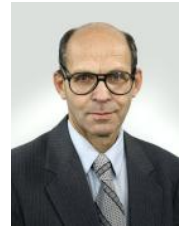

Valery Vodovozov is a professor of Tallinn University of Technology, Estonia, from 1997. He educated at the Technical University of Vologda and received his Candidate of Science degree and Associate Professor and Senior Researcher academic titles in Electrical Engineering from St. Petersburg Electrotechnical University, Russia, where he worked since 1976 till 2007 as an associate professor and a senior researcher. His teaching includes electric drives, electronics, and programming of electromechanical and human informational systems. His areas of expertise envelop electric drives and power electronics, application of object-oriented technologies in industry, and Web-based education. 